Relations industrielles

Industrial Relations

\title{
Carol JOBIN: Les relations du travail dans l'industrie de la construction. Montréal, Wilson \& Lafleur, 1989, 568 pp., ISBN 2-89127-085-1
}

\section{Jean Sexton}

Volume 44, numéro 2, 1989

URI : https://id.erudit.org/iderudit/050508ar

DOI : https://doi.org/10.7202/050508ar

Aller au sommaire du numéro

Éditeur(s)

Département des relations industrielles de l'Université Laval

ISSN

0034-379X (imprimé)

1703-8138 (numérique)

Découvrir la revue

Citer ce compte rendu

Sexton, J. (1989). Compte rendu de [Carol JOBIN: Les relations du travail dans l'industrie de la construction. Montréal, Wilson \& Lafleur, 1989, 568 pp., ISBN 2-89127-085-1]. Relations industrielles / Industrial Relations, 44(2), 473-473. https://doi.org/10.7202/050508ar

Tous droits réservés ㄷ Département des relations industrielles de l'Université Laval, 1989
Ce document est protégé par la loi sur le droit d'auteur. L’utilisation des services d’Érudit (y compris la reproduction) est assujettie à sa politique d'utilisation que vous pouvez consulter en ligne.

https://apropos.erudit.org/fr/usagers/politique-dutilisation/ 
Les relations du travail dans l'industrie de la construction, par Carol Jobin, Montréal, Wilson \& Lafleur Ltée, 1989, 568 pp., ISBN 2-89127-085-1

Les observateurs de la scène des relations du travail dans l'industrie québécoise de la construction dénoncent régulièrement le peu de documentation disponible sur ce sujet.

L'ouvrage que nous présente Carol Jobin comble de façon magistrale une partie de ce vide. En effet, cet instrument réunit et explique l'ensemble des textes législatifs et réglementaires régissant la construction au Québec au $1^{\text {er }}$ septembre 1988.

Ce livre comporte deux parties: l'auteur explique d'abord de façon fort détaillée les lois et règlements régissant les relations du travail dans l'industrie de la construction (175 pages). La deuxième partie reproduit intégralement le texte de ces lois et règlements (y compris le décret de la construction) dans trente-deux annexes différentes. Une table des matières très détaillée, un index thématique et un index par articles de lois et de règlements aideront le lecteur à s'y retrouver.

L'optique de la présente recension n'est pas juridique. Là n'est pas mon domaine. J'ai examiné cet ouvrage sous l'angle des relations du travail qui, comme on le sait, ne sont d'abord pas juridiques, et ce encore plus dans l'industrie de la construction.

Dans ce genre de travail, c'est souvent le premier chapitre qui est important puisque c'est là que l'auteur campe son sujet, explique sa méthodologie et présente les limites du travail qu'il présente. Dans un tel contexte, le premier chapitre de ce livre est décevant, surtout en raison de la trop timide mise en situation (section 1) que l'auteur présente. La nature de l'industrie, son évolution historique et les raisons de cette évolution, les parties en présence et leurs structures devraient occuper une place beaucoup plus importante permettant ainsi au lecteur de mieux situer et de mieux comprendre les particularités de la construction.

Vient ensuite un exposé des lois et règlements. Eu égard à la loi, l'auteur la présente non pas en suivant le plan de celle-ci mais plutôt par l'examen de seize thèmes particuliers. Cette approche est certes heureuse puisqu'elle permet de s'y retrouver beaucoup plus facilement. Ce faisant, l'auteur a décidé de limiter au strict minimum ce que l'on pourrait qualifier d'interprétations de textes. Il procède plutôt par dissection logique du texte de la loi. Suite à cet examen, l'auteur s'arrête aux recours que la lecture de la loi permet d'identifier.

En guise de conclusion, Carol Jobin démontre très clairement que la complexité, l'ambiguité et les contradictions internes de ce régime de relations du travail justifient pleinement la priorité souvent réclamée de s'attaquer à une réforme sérieuse de ce système.

Carol Jobin a sûrement fait oeuvre utile. Certes, tout travail est perfectible. Mais compte tenu de la complexité du sujet abordé et de la pénurie de documentation, surtout juridique, sur l'industrie de la construction au Québec, cet ouvrage en aidera plusieurs.

C'est un outil indispensable à toute personne intéressée ou impliquée dans l'industrie de la construction au Québec.

L'auteur sera sûrement appelé à reviser cet ouvrage vu les changements législatifs ou réglementaires relativement fréquents. De plus, le décret de la construction qu'il y présente est expiré depuis le 30 avril 1989. Cette nécessité de mise à jour presque régulière constitue certes un travail de bénédictin pour Carol Jobin. Mais son oeuvre étant si utile, espérons qu'il la poursuivra. Faudra-t-il que la prochaine édition soit à feuilles mobiles faciliant ainsi les revisions ultérieures?

Jean SEXTON 